\title{
Seguridad ampliacionista en el acuerdo de paz de Colombia (2016): seguridad humana, medio ambiente y paz territorial ${ }^{1}$
}

\section{Enlargementist security in the Colombian peace agreement (2016): human security, environment and territorial peace}

William Farfán Moreno*

Universidad Santo Tomás

\section{Resumen}

Una de las principales preocupaciones del Acuerdo final para la terminación del conflicto y la construcción de una paz estable y duradera (2016) es garantizar, mediante estrategias de seguridad, los derechos económicos, sociales, culturales, políticos y ambientales de las poblaciones y territorios que, especialmente,

${ }^{1}$ El presente artículo de reflexión, producto de investigación, hace parte del proyecto Crímenes ambientales y seguridad humana: Aportes para la comisión para el esclarecimiento de la verdad, la convivencia y la no repetición y el marco del Código Penal colombiano, financiado por la Escuela Superior de Guerra. Se encuentra en la fase de desarrollo conceptual y metodológico. " Doctor en Ciencia Política de la Universidad de Belgrano, Buenos Aires, magíster en Filosofía de la Universidad de los Andes, Bogotá y filósofo de la Universidad de La Salle. Investigador de la Escuela Superior de Guerra y docente investigador de la Universidad Santo Tomás, Bogotá-Colombia. ORCID: https://orcid.org/0000-0002-8198-2100. Google Académico https://scholar. google.es/citations?user=FNOADowAAAAJ\&hl=es 
se vieron afectados por el conflicto armado. Bajo este presupuesto, el objetivo del artículo es analizar los aspectos relacionados con la seguridad humana, medio ambiente y paz territorial en el Acuerdo final, con el fin de plantear algunas consideraciones acerca de su implementación. La metodología empleada fue el Análisis del Discurso Político (ADP) para sustentar que el Acuerdo final es un discurso político y, como tal, es susceptible de ser analizado empíricamente. La principal conclusión es la necesidad de implementar una política de seguridad en sentido amplio que tenga como centro la persona y las comunidades. Dicha seguridad se encuentra expresada en el punto de la reforma rural integral y, parcialmente, en el punto de la solución al problema del narcotráfico. Como conclusión, se plantea la necesidad de implementar dichos puntos con un enfoque de seguridad ampliada, especialmente, pero combinada con la seguridad tradicional limitadamente, sin que se traslapen para evitar nuevas violencias en los territorios.

\section{Palabras clave}

Consolidación de la paz; Seguridad humana; Medio ambiente natural; Acuerdo de paz; Conflicto armado.

\section{Abstract}

One of the main concerns of the Colombian Peace Agreement (2016) is to guarantee, through security strategies, all economic, social, cultural, political and environmental rights of peoples and territories that were especially affected by the armed conflict. Under this assumption, it is the goal of this article to analyze different aspects related to human security, environment and territorial peace contained within the agreement, in order to propose some considerations regarding its implementation. The methodology employed is Political Discourse Analysis, in order to sustain that the agreement constitutes a political discourse, and as such, it is susceptible of being analyzed empirically. The main finding is the need for implementing an expanded security policy that has as its center people and communities. This type of security is manifested in the chapter of integral rural reform, and partially, in the chapter of the solution to the drug trafficking problem. In conclusion, it is necessary to implement these chapters with an expanded security approach, but also combined with traditional security in a limited way, without overlapping each other, to avoid further violence in the territories.

\section{Keywords}

Peacebuilding; Human security; Natural environment; Peace agreements; Armed conflicts. 


\section{Introducción}

El documento del Acuerdo final para la terminación del conflicto y la construcción de una paz estable y duradera (de aquí en adelante Acuerdo final) firmado entre el Gobierno Santos y las Fuerzas Armadas Revolucionarias de Colombia, Ejército del Pueblo (FARC-EP), en noviembre de 2016 se ha convertido, desde su firma, en el centro de análisis de muchas investigaciones académicas. Una de las razones es que el proceso realizado por el Gobierno Santos ha sido el único que ha logrado construir y firmar, entre las partes enemigas, un documento final para ser implementado en un escenario de posconflicto.

Los objetivos del Acuerdo final fueron la terminación del conflicto armado y la construcción de una paz estable y duradera. En el aspecto de la construcción de la paz, según el documento, se encuentra una serie de variables para tener en cuenta durante la implementación de los acuerdos (posconflicto), entre ellas, la seguridad, ya sea física, política y jurídica. En el preámbulo del texto, se hizo énfasis en el reconocimiento de los derechos de la sociedad a una seguridad humana integral, dejando entrever que el horizonte del acuerdo y de la paz es una seguridad amplia y no restringida a la protección exclusiva del Estado: seguridad humana.

Bajo esta premisa, el objetivo del presente artículo es analizar los aspectos identificables sobre seguridad humana, medio ambiente y paz territorial en el Acuerdo final (2016) firmado entre el Gobierno colombiano y las FARC-EP, con el fin de plantear algunas consideraciones acerca de la implementación sobre estos puntos acordados.

Para el desarrollo del objetivo, se realiza una conceptualización de la seguridad humana y su relación con el medio ambiente y la paz territorial (marco teórico); posteriormente, se identifican y analizan los puntos sobre seguridad humana desde el enfoque de la seguridad ambiental y la paz territorial contenidas en el Acuerdo final mediante el Análisis del Discurso Político (metodología y resultados); seguidamente, a partir del análisis, se desarrollarán algunas conclusiones y consideraciones finales desde las acciones que el Estado ha implementado respecto a la triada seguridad humana, medio ambiente y paz territorial (análisis de resultados y conclusiones). 


\section{Discusiones preliminares: Acuerdo final, implementación y seguridad humana, medio ambiente y paz territorial}

Las Naciones Unidas con apoyo de Cooperación Alemana (2014), en el contexto del posacuerdo, realizaron un listado de municipios priorizados para la implementación de los acuerdos de paz desde el enfoque de paz territorial. Según el estudio, se deben priorizar 125 municipios que pertenecen a 17 departamentos de los 32 que conforman el territorio colombiano. De esos 125 municipios, el 37,6 \% tiene prioridad alta, y el resto prioridad media-alta. La prioridad alta se debe, en gran parte, a los impactos, no solo sobre el medio ambiente, sino sobre las poblaciones afectadas por las actividades económicas ilegales con el medio ambiente y por la presencia de actores armados.

Por otra parte, la Fundación Paz y Reconciliación (2015) logró también, desde un enfoque territorial y diferencial, priorizar los municipios donde se debieron diseñar las estrategias para la implementación luego de firmado el Acuerdo final en 2016. En total, son 281 los municipios del postconflicto en los que hay una presencia diferenciada de las FARC-EP del ELN, en los que se deberá aplicar el enfoque territorial del Acuerdo final prioritariamente. Por otra parte, existen 300 municipios donde hay presencia de economías ilegales y grupos surgidos luego de la desmovilización de las autodefensas; se puede decir que estos municipios se encuentran en una situación intermedia de intervención para la implementación.

Del informe de la Fundación Paz y Reconciliación (2015) se destacan dos conclusiones relacionadas con el impacto ambiental: primero, que de las 14 regiones donde las FARC-EP operan, 11 tienen una presencia importante de economías ilegales y, de las 7 donde está el ELN, 6 presentan economías ilegales basadas en cultivos ilícitos, minería o rentas ilegales urbanas; segundo, de los 281 municipios prioritarios, 88 se encuentran en vulnerabilidad extrema, 84 en vulnerabilidad alta y 109 en vulnerabilidad media. En los municipios de vulnerabilidad extrema se encuentran los municipios de 15 departamentos donde se desarrollan actividades económicas ilegales basadas en minería ilegal y cultivos ilícitos, destacándose Nariño (15 municipios), Chocó (14 municipios) y Antioquia (9 municipios), y que tienen una afectación directa sobre el medio ambiente y las comunidades.

Por otra parte, Rodríguez et al. (2017) priorizan 49 municipios donde se deben concentrar los esfuerzos de la implementación del Acuerdo final. Dichos municipios, ubicados mayoritariamente en la periferia del país, se caracterizan por la presencia de diferentes grupos armados ilegales, actividades como minería ilegal o cultivos de coca, por su situación de pobreza y desarrollo, y por la presencia débil o nula de las instituciones del Estado. 
Dichos estudios del 2014, 2015 y 2017 respectivamente, pueden contrastarse con los informes sobre los avances en los puntos relacionados con las subcategorías analizadas presentados tanto por el Instituto Kroc2 (2019), como en el Informe anual del Alto Comisionado de las Naciones Unidas para los Derechos Humanos (Naciones Unidas, 2020) respecto de la situación de los derechos humanos en Colombia. Para este artículo se tendrá en cuenta lo relacionado con el punto 1 (RRI) y el punto 4 (relacionado con el narcotráfico y los cultivos ilícitos).

Por una parte, el Instituto Kroc, en su tercer informe que va de 2016 a noviembre de 2018, respecto del punto de la RRI, afirma que de los 104 compromisos adquiridos, el $38 \%$ no ha iniciado, el $51 \%$ ha logrado un nivel mínimo de implementación, el $9 \%$ ha alcanzado un nivel intermedio de implementación y el $2 \%$ ha sido implementado completamente3. Entre los principales avances se destacan la creación del Fondo de Tierras para la formalización y adjudicación para campesinos y comunidades indígenas, la definición de las bases técnicas para precisar la nueva zonificación ambiental y delimitar la frontera agrícola, la creación del incentivo Pago por Servicios Ambientales, las estrategias técnicas para los planes de reforma rural integral y de sustitución de cultivos, la creación de los Centros de Diálogo Ambiental para tramitar los conflictos entre uso y vocación de la tierra y la formulación participativa de la zonificación ambiental.

Las principales dificultades, según el Instituto Kroc, son la lentitud en la implementación, el retraso legislativo, el incremento de la deforestación (en Amazonía)-que impide la estabilización de la frontera agrícola - y la falta de articulación entre los Programas de Desarrollo con Enfoque Territorial (PDET) con otros programas y planes territoriales, además de la preocupación de las comunidades por su estabilidad financiera.

Con respecto al punto 4, sobre la solución al problema de las drogas ilícitas, el Instituto Kroc afirma que de los 66 compromisos adquiridos, el $36 \%$ no ha iniciado, el $45 \%$ ha logrado un nivel mínimo de implementación, el $17 \%$ un nivel intermedio y el $2 \%$ ha sido implementado completamente. El avance más significativo ha sido el aumento del número de familias que han optado por la sustitución voluntaria de cultivos como una forma altamente efectiva para avanzar en la erradicación de coca y con muy bajos niveles de resiembra. Infortunadamente, esto contrasta con la principal dificultad: la incursión de organizaciones criminales que se disputan el control territorial en zonas de sustitución de cultivos. Por eso propone el Instituto un modelo de seguridad rural con participación de las comunidades que incluya la presencia integral de Estado.

Por otra parte, con respecto al Informe anual del Alto Comisionado de las Naciones Unidas para los Derechos Humanos (2020), durante 2019 hubo avances en restitución de tierras beneficiando a 6.647 familias. Sin embargo, en algunas regiones hubo dificultades debido a la presen-

${ }^{2}$ Los avances en términos técnicos de la implementación de los puntos acordados, según el Acuerdo final, estarán a cargo del Instituto Kroc de Estudios Internacionales de Paz de la Universidad de Notre Dame de los Estados Unidos.

${ }^{3}$ El Instituto Kroc (2019a) actualiza esta información a febrero de 2019. Los avances no son significativos, pues de lo implementado, el 51 \% ha logrado un nivel mínimo de implementación, el $8 \%$ ha alcanzado un nivel intermedio de implementación y el $3 \%$ ha sido implementado completamente. 
cia extendida de economías ilícitas sobre la tierra objeto de restitución. En cuanto a la pobreza multidimensional, se incrementó en un 1,8 \% afectando el acceso a varios derechos económicos, sociales y culturales, y agudizando la desigualdad con un índice de Gini de 0,51. El informe llama la atención sobre la brecha de desigualdad entre las zonas rurales y las zonas urbanas, que es uno de los problemas a superar del Acuerdo final:

\section{El porcentaje de personas viviendo en situación de pobreza multidimensional fue tres veces más alto en las zonas rura- les que en las zonas urbanas. El porcentaje de hogares sin acceso a agua potable en zona rural fue casi 16 veces más alto que en zonas urbanas y el analfabetismo en áreas rurales fue casi 4 veces más alto. Esto evidencia un incremento comparado con 2018. Los pueblos indígenas y las comunidades afrocolombianas, la mayoría de las cuales viven en zonas rurales, se ven desproporcionadamente afectados por dicha discriminación. (Naciones Unidas, 2020, p. 15)}

En cuanto a la RRI, Naciones Unidas (2020) destaca que en 2018 fueron formulados 16 PDET con buena participación comunitaria, incluyendo pueblos indígenas y comunidades afrocolombianas. Sin embargo, observa pocos avances y coordinación con otros programas igualmente importantes. En 2018 se aprobaron dos Planes Nacionales para la RRI, pero en 2019 no hubo ningún avance al respecto. Por último, afirma que para el año 2020 se redujo el presupuesto para las entidades responsables de la implementación de la RRI, un punto delicado porque todo este punto del Acuerdo final necesita una financiación robusta.

En el aspecto ambiental, el informe de UN (2020) destaca la ratificación del Convenio de Minamata sobre el Mercurio, pero denuncia la contaminación por mercurio en las cuencas de los ríos Apaporis, Atrato, Caquetá, Cauca y Nechí, afectando a los pueblos indígenas, comunidades afrocolombianas y campesinos. Otro caso sucedido en 2019 fue el de la represa Hidroituango, que generó impactos directos sobre los derechos al trabajo, al agua, a la alimentación, a un nivel de vida adecuado y a un ambiente sano de las comunidades. La solución generó impactos directos más negativos que los anteriores: como el río Cauca se secara, causando la muerte de peces, destruyendo cultivos y generando la desaparición de fuentes de agua, de nuevo las comunidades se vieron fuertemente afectadas. Por último, llama la atención sobre la erradicación de cultivos ilícitos en cuanto a que el Gobierno Nacional ha insistido, mediante proyecto de decreto del 30 de diciembre, en retomar el uso de glifosato, poniendo en riesgo la seguridad alimentaria, la salud y la privación de medios de subsistencia de las comunidades. Además, el informe vio con buenos ojos la erradicación voluntaria al indicar "que el 95\% de las familias que participan en el PNIS [Programa Nacional Integral de Sustitución de Cultivos de Uso Ilícito] cumplieron el requisito de erradicación voluntaria y sólo el $0.4 \%$ retornó a los cultivos de uso ilícito” (p. 18).

Estas discusiones preliminares mostraron, en perspectiva de Acuerdo final, la necesidad de priorizar municipios directamente afectados por el conflicto armado interno y donde la seguridad humana de personas, grupos societales y comunidades estaba en riesgo por la presencia de grupos armados y el impacto sobre el medio ambiente que estos podrían ocasionar. En este contexto surge la necesidad de identificar los aspectos relevantes de la seguridad humana, su relación con el medio ambiente y la paz territorial en el Acuerdo final. 


\section{La seguridad humana, medio ambiente y paz territorial en el Acuerdo de paz}

Respecto de la seguridad humana, en el Acuerdo final se mencionan elementos explícitamente relacionados con el desarrollo y que deben estar centrados en el buen vivir de la gente. Por ejemplo, en la Reforma Rural Integral (RRI) se estipula la necesidad de hacer una

Transformación estructural del campo, cerrando las brechas entre el campo y la ciudad y creando condiciones de bienestar y buen vivir para la población rural. La "Reforma Rural Integral" debe integrar las regiones, contribuir a erradicar la pobreza, promover la igualdad y asegurar el pleno disfrute de los derechos de la ciudadanía. (Mesa de Conversaciones, 2017, p. 7)

En otras palabras, cualquier iniciativa relacionada con la implementación de los acuerdos en el sector rural debe tener como centro de la seguridad el bienestar y buen vivir de las comunidades del campo.

En cuanto a la erradicación de la pobreza como forma de desarrollo, el Acuerdo final contempla que no debe ser visto solo desde el aspecto de ingresos de las familias, sino también en el aseguramiento de un acceso adecuado a servicios y bienes públicos. Vale la pena mencionar que este aspecto de la seguridad humana en el Acuerdo final (superación de la pobreza) es compatible con el Índice de la Pobreza Multidimensional (IPM) propuesta por la Universidad de Oxford (Alkire \& Santos, 2010), acogida por la PNUD (2010) e implementada por el Gobierno Santos en 2011 (Departamento Nacional de Planeación, 2011). En términos generales, el IPM es:

\footnotetext{
The MPI is an index of acute multidimensional poverty. It reflects deprivations in very rudimentary services and core human functionings for people across 104 countries. Although deeply constrained by data limitations, the MPI reveals a different pattern of poverty than income poverty, as it illuminates a different set of deprivations. The MPI has three dimensions: health, education, and standard of living. (Alkire \& Santos, 2010, p. 7).
}

Se hace uso del IPM, primero, porque tiene una relación estrecha con el desarrollo humano de cada país, segundo, porque tiene en cuenta variables que anteriormente no se tenían en cuenta para conocer la situación real de privaciones tanto en ciudades capitales como en zonas rurales (salud, educación y nivel de vida), y tercero, porque es el índice que oficialmente se utiliza desde el 2010 en Colombia para medir el nivel de pobreza o privaciones. Si el Acuerdo final tiene como uno de sus objetivos la erradicación de la pobreza, una forma de conocer estos avances es mediante el IPM, ya que no propone un método de medición porque ya existe en Colombia.

Siguiendo con la línea de la seguridad humana -muchos de cuyos elementos son compatibles con el IPM-, existen otros aspectos que tiene en cuenta el documento del Acuerdo final, como son la promoción de educación formal (básica y media, técnica y tecnológica, universitaria) y educación para el trabajo y el desarrollo humano; de vivienda; de cultura, recreación y deporte; 
de protección y recuperación del medio ambiente. En general, como lo afirma el mismo Acuerdo final, la seguridad debe entenderse como un valor democrático y bajo la perspectiva del humanismo debe inspirar las formas de acción por parte del Estado.

Pero también hay otra variable relacionada con la seguridad humana, que el documento expresa claramente, y es la seguridad ambiental, pues se considera que los daños ambientales afectan el bien vivir de las comunidades. Por ello, en el Acuerdo final se propone buscar mecanismos o acciones que permitan mitigar cualquier tipo de daño ambiental en zonas de especial interés ambiental, ecosistemas frágiles e hidrografía vulnerable y para la recuperación de los bosques. Esta idea se conecta, a su vez, con el concepto de paz territorial desarrollado por Jaramillo (2013), entendido como un conjunto de estrategias que garanticen los derechos fundamentales y humanos, promuevan la economía campesina con un enfoque territorial, la participación ciudadana, la presencia del Estado y la protección del medio ambiente con el único fin de lograr la paz estable y duradera.

Así pues, para proteger a las comunidades, el Acuerdo final pone en consideración una triada inseparable sobre seguridad para los propósitos de la paz estable y duradera: seguridad humana, ambiental y paz territorial. El primero, promoviendo los bienes necesarios para la erradicación de la pobreza y la desigualdad social; el segundo, fortaleciendo los proyectos ambientales sostenibles y productivos; el tercero, protegiendo los territorios y sus comunidades con un enfoque diferencial. Por lo tanto, en el Acuerdo final se promueve esta idea de seguridad humana relacionada con el ambiente y los territorios.

\section{Marco teórico}

\section{Seguridad ampliacionista: seguridad humana, medio ambiente y paz territorial}

Para poder realizar un trabajo conceptual que relacione seguridad humana/ambiente, es necesario desarrollar primero la relación seguridad/ambiente, ya que la seguridad humana es tan solo uno de tantos tipos de seguridad que se han trabajado desde la academia.

El concepto de seguridad, como lo sostienen Oswald-Spring y Günter-Brauch (2009), ha adquirido múltiples connotaciones desde las ciencias políticas, la sociología y la economía, adquiriendo un carácter interdisciplinar. No en vano, el entonces Secretario General de la Organización 
de las Naciones Unidas (UN), Javier Pérez de Cuellar, presentó un informe sobre "los conceptos de seguridad" en donde afirmó que estos han evolucionado en respuesta a las necesidades de la misma seguridad nacional y los cambios circunstanciales en los ámbitos políticos, militares y económicos (United Nations, 1986).

Con el informe de la United Nations (UN) se pueden sintetizar algunas características que ha adquirido la seguridad: todas las naciones tienen derecho a la seguridad; la fuerza militar con fines de autodefensa es un instrumento legítimo de política nacional; la seguridad debe entenderse en términos comprehensivos y reconocer la interdependencia de los factores políticos, económicos, sociales, geográficos y tecnológicos; los problemas de seguridad y las amenazas conciernen a todas las naciones, por tanto es un deber y un derecho de ellas buscar soluciones constructivas; ningún factor debido a la diversidad política, social, étnica, ideológicas, entre otras, podrá ser un obstáculo para la cooperación internacional para la paz y la seguridad; el desarme y limitación de armas es la tarea más urgente de la comunidad internacional para la cooperación de la paz y seguridad internacional (UN, 1986). Estas connotaciones sobre la seguridad dejan entrever una idea de ella en un sentido tradicional, en el que se centra más en la protección de los Estados que en las personas.

Por otro lado, Brauch-Günter (2009) analiza el concepto de seguridad a partir de los estudios sobre el orden internacional, desde el Tratado de Versalles -que introdujo el concepto de "seguridad colectiva"-, pasando por la Segunda Guerra Mundial, hasta la Guerra Fría - con la implementación de "la seguridad nacional" para darle un papel primordial a los Estados Unidos en el orden internacional-, hasta el orden posterior de los años noventa con la pos Guerra Fría -en que el concepto de seguridad se amplió a otros sectores distintos al militar, como la seguridad "humana" y "ambiental"-. A partir de este estudio realizado por Brauch y de acuerdo con el devenir del orden internacional, varios autores han complementado este trabajo mediante calificativos al concepto de seguridad, como seguridad objetiva y subjetiva (Weaver, 2009; Wolfers, 1962), seguridad colectiva e individual (Waever, 2009) y seguridad en sentido tradicional y en sentido ampliacionista (Brauch-Günter, 2009; Albrecht, 2009).

La seguridad objetiva responde a la pregunta de qué tan amenazado se encuentra alguien, y la subjetiva a qué tanto alguien percibe o interpreta que se encuentra amenazado (Waever, 2009). La primera habla de amenazas reales y la segunda de las precepciones sobre amenazas. Wolfers (1962), por otro lado, define la seguridad, en un sentido objetivo, como la ausencia de amenazas a los valores adquiridos, y en un sentido subjetivo, como la ausencia del miedo a que dichos valores sean atacados.

Respecto a la seguridad individual y la seguridad colectiva, ambas tienen que ver con la evolución del Estado. La seguridad individual se desarrolla en el contexto del Estado liberal moderno cuyo fin es la protección de los derechos individuales. El papel único del Estado es garantizar la 
seguridad a los individuos para que gocen de sus derechos y libertades individuales, y contrasta con la seguridad colectiva, en cuanto se refiere a la protección de los intereses nacionales y el statuo quo de grupos dominantes para mantener la paz. Se contempla, de este mismo modo, la seguridad a grupos societales o comunidades (Waever, 2009).

Por último, la seguridad, también es concebida desde dos sentidos: en el sentido amplio y en el sentido tradicional. Autores como Buzan, Weaver y Wilde en Security: A New Framework for Analysis (1998) consideran que la seguridad debe ampliarse a las dimensiones económicas y ambientales, mientras que los tradicionalistas, como Walt, Chipman, Gray y Dorff se enfocan en la primacía de un concepto de seguridad militar (citados en Brauch, 2009, p. 184). Albrecht y Brauch-Günter (2009, p. 364), citando a Elise Boulding, se refieren a este debate entre los tradicionalistas y los ampliacionistas:

\begin{abstract}
Si el concepto de seguridad se estira de tal manera que signifique cualquier cosa, ¿no se corre el riesgo de que no signifique nada? El debate en esta sección, en parte es semántico. Ninguno de los autores que usan la definición limitada de seguridad -por ejemplo, seguridad ante un ataque- negaría que existen muchas otras amenazas al bienestar humano además de las amenazas militares. Lo importante es separar la estrategia de guerra, de la multitud de amenazas que enfrenta la humanidad hoy, e incluir las de destrucción ecológica y devastación económica. Los autores que defienden una definición más amplia basan sus análisis en un modelo de interconexión de variables sociales y físicas en un marco de sistema integral, que sus colegas no niegan, y de donde todos están de acuerdo en que la verdadera tarea es eliminar la destrucción en todas sus formas y aumentar el nivel de cooperación humana y de conciencia ambiental en la medida de lo posible. (p. 364)
\end{abstract}

Por un lado, si los tradicionalistas consideran que la seguridad debe limitarse a la defensa haciendo uso del aparato militar, podrían dejar de considerar otros tipos de amenazas para la humanidad; por el otro, si los ampliacionistas extienden, amplían y profundizan el concepto de seguridad a otros sectores (ambiental, económico, por ejemplo), se correría el riesgo de ampliar el factor militar hasta el punto tal de militarizar la seguridad ambiental o económica. Es decir, la línea que separa a los tradicionalistas de los ampliacionistas es muy delgada. Albercht y BrauchGünter (2009) lo ponen en estos términos: “definir los asuntos ambientales en términos de riesgos de seguridad es en sí una operación riesgosa (...) podemos terminar contribuyendo más a la militarización de las políticas ambientales que a la desmilitarización de las políticas de seguridad" (p. 366).

Así pues, se encuentra en este tipo de debates los nuevos discursos acerca del concepto de seguridad en el que la militarización y los nuevos problemas sociales contemporáneos se traslapan ocasionando dificultades en el momento de tomar decisiones gubernamentales en torno a la seguridad.

Con el fin de la Guerra Fría, muchas de las reflexiones sobre la seguridad las aplicaron los gobiernos en sus conceptos extendidos y ampliados. Al contemplar la existencia de otros tipos de amenazas distintos a los llamados Estados comunistas, se considera también que las formas de violencia se han ampliado desde los conflictos intra-estatales hasta los transnacionales, tales 
como el terrorismo, la criminalidad, la inseguridad pública, el narcotráfico, entre otros. Por ello, la seguridad se ha ampliado hasta incluir la protección de la gente y sus derechos frente a este tipo de desórdenes, apuntando más a la seguridad interna o doméstica:

Mientras que el sistema del Estado-nación de Westfalia dominó el análisis de seguridad durante la Guerra Fría, a partir de 1990 aparecieron nuevos actores: desde abajo (insurgentes, comunidades étnicas y regionales) y desde arriba del Estado (corporaciones multinacionales e instituciones multilaterales), así como actores transnacionales (terroristas, redes criminales). (Albrecht y Brauch-Günter, 2009, p. 366)

Fue durante la Guerra Fría en que se desarrollaron las principales tradiciones científicas sobre la seguridad: el realismo, el idealismo y el racionalismo en el contexto de la bipolaridad del orden internacional, es decir, que estuvo permeada por la cosmovisión occidental anglosajona. El realismo contemporáneo tiene sus raíces en autores como Tucídides, con su obra Historia de la guerra del Peloponeso (s.f.), Maquiavelo con El Príncipe (1532), Hobbes con El Leviatán (1651) y Rousseau con El Estado de guerra (1750). Por otra parte, el idealismo tiene su referente en Grocio, con Del derecho de la guerra y de la paz (1925) y Prolegomena to the Law of War and Peace (1975), pero principalmente en Immanuel Kant con su ensayo Hacia la paz perpetua (1795), puesto que logró poner en tela de juicio la idea de paz de Westfalia según la cual la guerra es el medio para la resolución de los conflictos interestatales.

Sin embargo, existen lecturas contemporáneas de escuelas europeas que no se remiten al ámbito académico norteamericano, como la Escuela de Copenhague. Esta surgió como propuesta alternativa a los realistas, liberales y racionalistas a quienes se les criticaba por su actitud observante y no participante de la realidad internacional. La Escuela de Copenhague, que tiene como representantes a Buzan y Weaver (2003), tiene una visión amplia del concepto de seguridad, no limitándolo exclusivamente a las amenazas militares, sino extendiéndolo a otros aspectos provenientes de ámbitos sociales, económicos o ambientales. Estos autores consideran que dicha ampliación del concepto ha llevado a los procesos de securitización dados mediante los "actos discursivos".

Dentro de esta gran área de los estudios de la seguridad alternativos a los "racionalistas" se encuentra los reflectivistas, llamados así porque consideran que se requiere de nuevos conceptos que "reflejen" la situación contemporánea para comprenderla mejor (Bartolomé, 2006). Existen dos corrientes de los reflectivistas: los constructivistas y los postmodernistas. En cuanto a los primeros, sostienen que para la interpretación de la realidad no era suficiente la observación, pues consideran que la realidad se construye desde lo lingüístico y sociológico de tal forma que esta realidad se encuentra determinada por el discurso y el lenguaje. De ahí que se les llame constructivistas. Por otra parte, los posmodernistas rechazan, al igual que los constructivistas, la idea de territorialidad y soberanía como argumentos para justificar el papel del Estado como protagonista del sistema internacional. Los posmodernistas consideran que existen otras variables más reales 
y menos absolutas como la geografía, pues es en los espacios y territorios reales y no abstractos donde se despliegan y materializan las amenazas. Aquí, una vez más, se evidencia el rechazo de los reflectivistas al racionalismo de los realistas y liberales.

Es la Escuela de Copenhague quien expone una dimensión sobre seguridad diferente a las connotaciones tradicionales que centraban los objetos referentes de securitización en los Estados. Las nuevas amenazas de finales del siglo XX y comienzos de XXI, como el cambio climático y la economía, ponen en el escenario una idea amplia de seguridad centrada, no en el Estado, sino en la persona. De este modo es que entra el concepto de seguridad humana en los estudios empíricos y analíticos.

La seguridad humana es una noción que tiene como tema principal el desarrollo humano y está centrada en las comunidades humanas y no en los Estados. Fue empleada de forma categórica en el informe del PNUD en los años 1993 y 1994, abarcando 2 aspectos y 7 categorías (Bartolomé, 2006). En cuanto a los aspectos, el primero se refiere a la seguridad contra amenazas crónicas como el hambre, las enfermedades y las libertades individuales; y el segundo se remite a las amenazas propias de la vida cotidiana de los individuos en los escenarios familiar, laboral y comunitario. Las 7 categorías, por su parte, son la seguridad económica, alimentaria, en salud, ambiental, comunitaria, política y personal. Como se puede observar, la seguridad ambiental es una de las categorías insoslayables de la seguridad humana y, por supuesto, uno de los objetos de estudio sobre investigaciones relacionadas con los conflictos armados.

Solo hasta finales de la Guerra Fría, el estudiar los impactos sobre el medio ambiente dejados por los conflictos armados, y sus consecuencias sobre las poblaciones, se ha convertido en objeto de interés para las investigaciones, pues estos son considerados como amenazas latentes para las comunidades, ya sea porque el medio ambiente ha sido objetivo militar de los grupos armados o porque han sido daños colaterales del conflicto bélico.

El paso hacia una conceptualización de la seguridad humana y ambiental, como lo expone Brauch-Günter (2009), que se ha venido desarrollando desde el 2004, introdujo en las diferentes investigaciones las nuevas amenazas del siglo XXI, tales como el cambio climático, la desertificación, la erosión de suelos, la pérdida de biodiversidad, la desforestación, la escasez de agua y degradación del medio ambiente, con sus impactos y resultados societales como migración forzada, crisis económicas, emergencias complejas, prevención y mitigación de conflictos y su resolución. Estas situaciones han puesto a los individuos a elegir entre seguir viviendo en zonas donde están presentes las amenazas de tipo ambiental y humano, o emigrar a campos de refugiados o centros urbanos de sus propias naciones u otros países para sobrevivir -lo que, afirmaría Brauch-Günter (2009), los pone ante un "dilema de supervivencia"-. 
Según la The United Nations Environment Programme (UNEP), en los últimos 20 años, al menos 18 guerras civiles e internas han sido financiadas gracias a la explotación de los recursos naturales. El estudio muestra los casos recientes en que los recursos naturales se convierten en patrocinadores de los conflictos internos, de cuyos países se destacan dos latinoamericanos: Perú (1980-1985), mediante la coca, y Colombia (1984-actualmente), con los recursos como petróleo, oro, coca, madera y esmeraldas. Según el estudio, el aprovechamiento económico que generan los recursos naturales y que benefician a individuos o diferentes grupos, puede ser un obstáculo determinante para superar los conflictos armados, facilitar los esfuerzos de paz e, incluso, lograr la reconciliación nacional luego de acuerdos de paz:

\begin{abstract}
Economic incentives related to the presence of valuable natural resources can hinder the resolution of conflict and complicate peace efforts. As the prospect of a peace agreement appears closer, individuals or splinter groups who stand to lose access to the revenues gained from resource exploitation can act to spoil peacemaking efforts. Indeed, real or perceived risks of how peace may alter access to and regulation of natural resources in ways that damage some actors' interests can be a major impediment. At the same time, natural resources can also undermine genuine political reintegration and reconciliation even after a peace agreement is in place, by providing economic incentives that reinforce political divisions. (UNEP, 2009, p. 11)
\end{abstract}

Según la UNEP (2009), el ambiente y la explotación de sus recursos naturales en un conflicto armado ha sido o una estrategia de guerra de los grupos implicados y por eso ha sido un objetivo militar, o sus daños han sido colaterales. Por otra parte, Estrada (2016), al explicar uno de los puntos del Acuerdo final menciona la nueva política de seguridad que allí se expone:

\footnotetext{
Una nueva comprensión de la política de seguridad que deje atrás las concepciones centradas en el control del orden público y en el combate al enemigo interno, para privilegiar en su lugar un enfoque integral en el que el dominio del Estado no sea reducido a la llegada o presencia de sus aparatos coercitivos, sino que además de la asunción de funciones sociales, se propicien procesos de construcción social que atiendan la historicidad concreta de la relación social territorial. Las elaboraciones de Naciones Unidas acerca de la seguridad humana resultan de particular pertinencia para el diseño de una política de seguridad para el "Fin del conflicto". Igualmente, las experiencias de autorregulación en comunidad, que nos indican que es posible pensar en formas no armadas de la seguridad, como en el caso de las guardias indígenas y más recientemente de las guardias campesinas. (Estrada, 2016, p. 98)
}

En el Acuerdo final, la seguridad en sentido amplio, el aspecto ambiental, se convierte en un punto insoslayable cuando de la seguridad humana centrada en la persona se habla, para concebir el bienestar y buen vivir de las comunidades. No obstante, cuando se trata de un conflicto armado como el colombiano, en el que lo territorial ha sido uno de los factores principales de la violencia (CNMH, 2016), obliga a hablar de lo que Jaramillo (2013) denominó paz territorial. Este concepto surge en el contexto del proceso de paz en Colombia, por lo que se puede decir que es un concepto sui géneris. En términos generales, como veremos en el siguiente apartado, el fin del conflicto tiene como finalidad garantizar los derechos fundamentales y humanos de todos los individuos que, en época de violencia, les fueron arrebatados, coartados o eliminados en varios territorios donde la presencia del Estado era débil o nula, y donde quienes hacían las veces de autoridad eran los grupos armados ilegales. Es en este contexto que Jaramillo (2013) afirma: 
Lo que me interesa resaltar es que tenemos que aprovechar el momento de la paz para alinear los incentivos y desarrollar las instituciones en el territorio que con el tiempo van a hacer valer los derechos de todos por igual. Para avanzar en esa dirección, hay que complementar el enfoque de derechos con un enfoque territorial. Primero porque el conflicto ha afectado más a unos territorios que a otros. Y porque ese cambio no se va a lograr si no se articulan los esfuerzos y se moviliza a la población en esos territorios alrededor de la paz. Eso es lo que llamo la paz territorial (p. 1)

Este texto de Jaramillo fue publicado en 2013 y, como veremos, en el Acuerdo final se encontrarán algunos puntos explícitos e implícitos sobre paz territorial. Se infiere, entonces, que el territorio no solamente se entiende como un espacio exclusivo para sector productivo o para el aprovechamiento de los recursos naturales (González, 2015), sino también como el espacio donde existe un conjunto de entramados socioeconómicos y políticos que fue afectado por el conflicto armado y que el Acuerdo final tiene en cuenta para la construcción de la paz estable y duradera con la participación ciudadana y la presencia de las instituciones políticas y sociales del Estado: "esta propuesta de paz territorial da pistas para un diálogo más amplio que el de las zonas rojas y permite explorar esa campaña de planeación participativa y de ejecución compartida o autónoma que reclaman comunidades campesinas y étnicas" (González, 2015, p. 10).

La paz territorial, por tanto, haría parte de la seguridad en sentido ampliacionista, puesto que cuando en el Acuerdo final se habla de la reforma rural -primer punto de lo acordado-, se la considera como un conjunto de estrategias para apoyar la economía campesina sin desdeñar de la protección y el cuidado del medio ambiente, lo que pone en el centro a la persona y las comunidades.

De acuerdo con lo anterior, para fines del análisis se va a identificar que la seguridad humana y la protección del medio ambiente son subcategorías de análisis de la categoría "seguridad ampliacionista". La idea, a continuación, es analizar qué puntos particulares del texto del Acuerdo final relaciona la seguridad humana con el medio ambiente a partir de estas categorías de análisis.

\section{Metodologia}

El análisis y evidencia de la investigación se realizó mediante el método del Análisis del Discurso Político (ADP) (Van Dijk, 1999) que hace parte de los estudios del discurso (Fairclough, 1995; van Dijk, 1999, 1999a, 2003) cuyo corpus para su análisis es el Acuerdo final Para ello, se parte de la idea de que el documento de estudio (Acuerdo final) es un discurso político porque hace parte del dominio político y, en consecuencia, es susceptible de ser analizado. El objetivo de aplicar el ADP fue analizar las categorías de seguridad humana, medio ambiente y paz territorial como subcategorías de análisis que hacen parte de la seguridad en sentido ampliacionista con el fin de plantear algunas consideraciones acerca de la implementación sobre estos puntos acordados. 
Van Dijk (1999) considera que, centrándose en la naturaleza de las acciones o prácticas producidas por el texto político o del acto, se delimita el objeto de estudio del ADP. Es de esta manera como toma relevancia el contexto para delimitar la categorización del discurso como "político"; por lo tanto, el discurso político se delimitará al acto de habla o al texto político realizado por políticos en contextos políticos que tengan afectación en la comunidad.

El lingüista holandés (1999) define unas características, desde lo abstracto a lo concreto, de las estructuras y de los procesos del dominio político, tanto del texto como del discurso políticos, que son compatibles con el texto del Acuerdo final:

Tabla 1

Características del Acuerdo final como discurso político

\begin{tabular}{|c|c|}
\hline Características & Discurso Acuerdo final \\
\hline Sistema & La democracia \\
\hline Institución & Ejecutivo \\
\hline Valores e ideologías & Fin del conflicto armado, paz, reconciliación, justicia \\
\hline Organizaciones & $\begin{array}{l}\text { Comisión Histórica del Conflicto y sus Víctimas. Mesas de participación } \\
\text { ciudadana. Distintas organizaciones de la sociedad civil. Académicos }\end{array}$ \\
\hline Actores políticos & $\begin{array}{l}\text { Negociadores del proceso de paz, tanto del gobierno (Comisionado de paz y } \\
\text { delegados) como de las FARC (voceros). Países acompañantes del proceso de } \\
\text { paz: República de Chile y República Bolivariana de Venezuela. Países garantes: } \\
\text { Reino de Noruega y República de Cuba }\end{array}$ \\
\hline Relaciones políticas & Enemigos del conflicto armado y partes en las mesas de negociación \\
\hline Proceso político & Proceso de paz entre Gobierno Santos y FARC \\
\hline Acción política & Fin del conflicto armado y construcción de una paz estable y duradera \\
\hline Cogniciones políticas & $\begin{array}{l}\text { Las actitudes sobre el proceso de paz y el conflicto armado (justicia, desarme, } \\
\text { desmovilización, víctimas, participación política, narcotráfico, tierras, seguridad) }\end{array}$ \\
\hline Dominio & La política \\
\hline
\end{tabular}

Fuente: elaboración propia.

Como lo afirma el lingüista holandés, el ADP es un método útil para el análisis de un texto entendido como discurso político cuando no puede ser analizado mediante otros instrumentos de análisis:

EI ADP únicamente puede ser aceptado por los cientistas políticos si tiene algo para ofrecer, preferiblemente si es algo que aquellos no podrían (conseguir) saber -por lo menos no del todo- mediante otros métodos, como las encuestas de opinión, la observación participante 0 el análisis de contenido. Y es un modo amplio que va desde observar el análisis del discurso no meramente como un "método", como el análisis de contenido, sino como una nueva (también teórica) disciplina interrelacionada con su propia perspectiva, una disciplina donde la ciencia política está involucrada. (Van Dijk, 999, p. 64)

Definido el Acuerdo final como discurso político, a continuación, se va a realizar el análisis a partir de la categoría "seguridad en sentido ampliacionista" con sus subcategorías "seguridad humana", "medio ambiente" y "paz territorial” mediante el uso de una matriz de análisis. 
Finalmente, las fases metodológicas del presente trabajo son 4 (ver Gráfica 1): en la fase 1 se identifican los códigos que se hallan en el corpus (Acuerdo final) relacionados con la seguridad humana como seguridad social, seguridad, seguridad con enfoque territorial y diferencial, seguridad alimentaria, entre otros que sirvieron para codificar el corpus mediante la herramienta Atlas. Ti. En la fase 2, dichos códigos se agrupan en familias que fueron inferidas en el marco teórico. En este caso, son las 3 categorías principales de análisis: seguridad humana, medio ambiente y paz territorial. En la fase 3 se realiza el análisis de dichas familias para, teniendo en cuenta el ADP, finalizar en la fase 4 con las conclusiones y recomendaciones.

Figura 1. Fases metodológicas

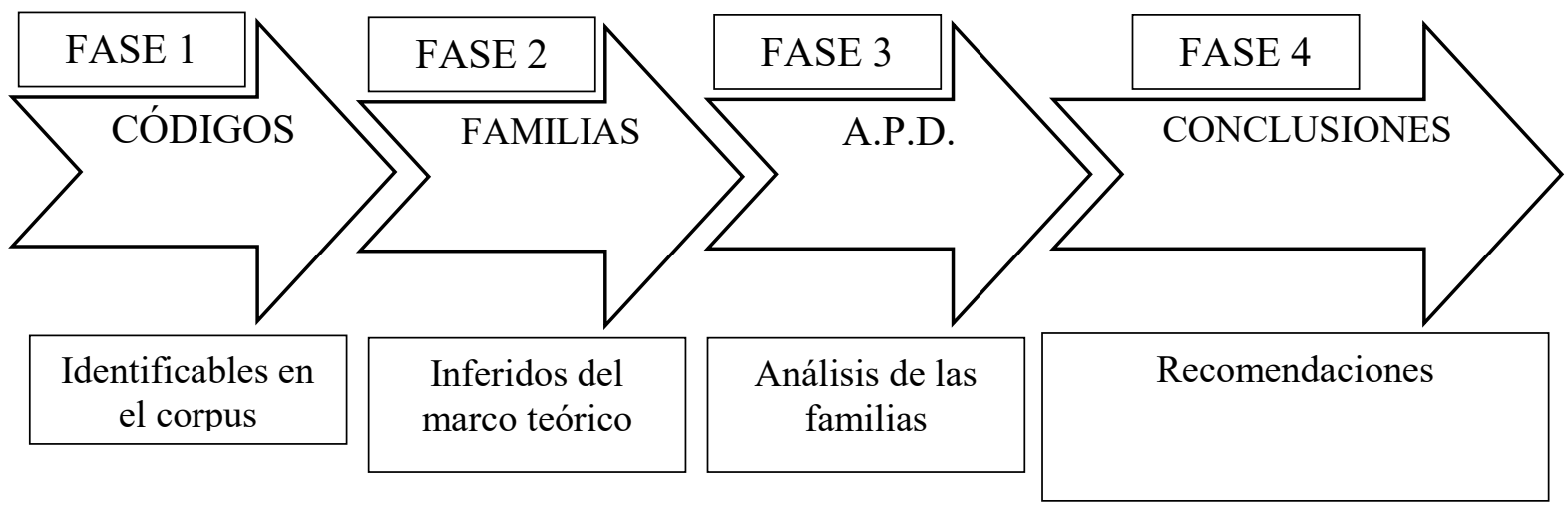

\section{Resultados. Análisis de seguridad humana, medio ambiente y paz territorial identificables en el Acuerdo final}

En el preámbulo del Acuerdo final aparecen tanto el concepto de "seguridad humana" como el término "medio ambiente" solo una vez respectivamente. En tanto preámbulo, denota como fuente para poder aplicar una interpretación de cualquiera de los puntos del acuerdo. Esto quiere decir que para interpretar cualquiera de ellos ante una posible ambigüedad en su implementación se debe acudir al preámbulo. En este caso particular, cuando se trata de la seguridad y el medio ambiente, se afirma: "Reconociendo los derechos de la sociedad a una seguridad humana integral con participación de las autoridades civiles" (Acuerdo final, 2016, p. 3).

Atentos a que la nueva visión de una Colombia en paz permita alcanzar una sociedad sostenible, unida en la diversidad, fundada no solo en el culto de los derechos humanos sino en la tolerancia mutua, en la protección del medio ambiente, en el respeto a la naturaleza, sus recursos renovables y no renovables y su biodiversidad. (Acuerdo final, 2016, p. 3) 
En las citas anteriores se logra entrever claramente que algunos de los horizontes filosóficos y políticos del acuerdo de paz son la seguridad humana, la protección del medio ambiente y la paz territorial. Respecto al primero, como ya se mencionó en el abordaje teórico, es una seguridad centrada en la persona y bajo la perspectiva del "humanismo" (Mesa de conversaciones, 2017, p. 39); respecto al segundo, la protección al medio ambiente, como el respeto a la naturaleza, sus recursos renovables y no renovables y su biodiversidad, implica una garantía insoslayable de la seguridad humana (seguridad en el sentido amplio), fundamentada en la tesis sobre el medio ambiente como una víctima del conflicto armado (Botche et al., 2010; Londoño y Martínez, 2015); por último, la paz territorial se refiere al conjunto de estrategias que deben diseñarse en la implementación para garantizar derechos sociales, económicos, culturales y ambientales de las poblaciones con un enfoque territorial y diferencial (Jaramillo, 2013).

Esta triada (seguridad humana, medio ambiente y paz territorial), como se mencionó anteriormente, son categorías que se encuentran de forma explícita e implícita en el texto y hacen parte de la "seguridad tradicional en sentido ampliacionista". Es de esta manera como se va a realizar el ADP en torno a este enfoque de la seguridad teniendo en cuenta las 3 categorías de análisis.

\section{Categoría de análisis: medio ambiente}

Como se mencionó en el abordaje teórico, el objetivo de la seguridad ampliacionista es, como su nombre lo indica, ampliar las dimensiones de seguridad a los temas económicos y ambientales con el fin de proteger a las personas o comunidades. En el Acuerdo final (2016) se propone buscar mecanismos o acciones que permitan mitigar cualquier tipo de daño ambiental "en zonas de especial interés ambiental, ecosistemas frágiles e hidrografía vulnerable y para la recuperación de los bosques" (p. 114).

En el discurso político del Acuerdo final, varias citas relacionan a la protección del medio ambiente con el sector rural, específicamente, con el sector agrícola. Apunta a que, en primera medida, se promoverán iniciativas rurales y productivas que, además de proteger el medio ambiente, también deberán preocuparse por la recuperación de zonas afectadas o potencialmente amenazadas por la actividad agraria.

Del mismo modo, llama la atención la necesidad de proteger el medio ambiente cuando de sustituir o erradicar los cultivos ilícitos se trata. El negocio del narcotráfico ha sido uno de los factores que ha permitido a los grupos armados ilegales financiar la guerra, dejando consecuencias humanas y ambientales. En el marco del acuerdo se plantea anular el narcotráfico y, para empezar, se deben erradicar los cultivos ilícitos. Para ello, se prioriza la erradicación manual y, en caso de 
ser necesario, incluir la aspersión siempre y cuando se respeten los derechos humanos, el medio ambiente, la salud y el buen vivir. En el caso de la aspersión, que en la mayoría de las veces se hace con el agroquímico del glifosato, y que ya ha sido empleado en Colombia, suena paradójico utilizar este mecanismo y, a la vez, proteger el medio ambiente y la salud de las comunidades. Este es un punto que debe discutirse ampliamente con investigaciones científicas, pero, de entrada, este aspecto iría en contra de esa seguridad en sentido amplio y se reduce a solucionar un problema complejo de una manera restrictiva.

Otro aspecto para destacar es la protección del medio ambiente como una forma de atender o garantizar los derechos fundamentales e integrales de las comunidades. Entre ellos está que un medio ambiente protegido y adecuado promueva el bienestar y buen vivir de las personas.

Por último, se destaca la idea de que el fin del conflicto permitirá garantizar la protección del medio ambiente mediante responsabilidades concretas como cuidar de no dañar el medio ambiente, realizar acciones para la mitigación de los daños ambientales en zonas de especial interés ambiental, ecosistemas frágiles e hidrografía vulnerable y para la recuperación de los bosques.

\section{Categoría de análisis: seguridad humana}

La seguridad humana hace parte de la seguridad en sentido ampliacionista puesto que apunta a los temas de desarrollo y está centrada en la gente más que en los Estados. En el Acuerdo final se menciona la necesidad de reconocer los derechos de la sociedad a una seguridad humana integral. En el texto se entiende la seguridad como valor democrático que debe inspirar la actuación del Estado bajo la perspectiva del humanismo. Esta comprensión de seguridad da cabida para repensarse cómo históricamente se ha desarrollado la seguridad en Colombia, desde la llamada Doctrina de Seguridad Nacional hasta la seguridad basada en la lucha contra el terrorismo, centradas en la protección del Estado y no en la persona. Con el Acuerdo final la seguridad toma la connotación ampliacionista cuyo objeto referente de securitización son la persona y las comunidades, característico de la seguridad humana. En este sentido, la seguridad no es vista como un elemento restrictivo de la promoción del desarrollo y el bienestar, sino como un elemento que securitizar los derechos sociales, económicos e individuales de personas, grupos societales y comunidades. Por ello, en el Acuerdo final se pretende "articular las medidas de seguridad con las medidas de desarrollo y bienestar individuales y colectivas contempladas en el presente acuerdo, y que adopta un enfoque diferencial y de género" (Acuerdo final, 2016, p. 38). 
Dentro de los temas de desarrollo centrado en la gente, en la "Reforma Rural Integral" (RRI) se estipula la necesidad de hacer una transformación estructural del campo que permita acabar con la brecha entre lo rural y la ciudad, con el fin de crear condiciones de bienestar y buen vivir para la población rural. Por otra parte, con la RRI se pretende erradicar la pobreza, promover la igualdad y asegurar el disfrute pleno de los derechos de la ciudadanía.

En cuanto a la superación de la pobreza como forma de desarrollo, el acuerdo contempla esto no solo en el aspecto de ingresos de las familias, sino también en el aseguramiento de un acceso adecuado a servicios y bienes públicos. El acuerdo entiende el desarrollo social como "(...) salud, educación, vivienda, erradicación de la pobreza" (Acuerdo final, 2016, p. 25).

\section{La superación de la pobreza no se logra simplemente mejorando el ingreso de las familias, sino asegurando que niños, niñas, mujeres y hombres tengan acceso adecuado a servicios y bienes públicos. Esa es la base de una vida digna. Por eso la superación de la pobreza en el campo depende, ante todo, de la acción conjunta de los planes nacionales para la Reforma Rural Integral. (Acuerdo final, 2016, p. 23)}

En este aspecto se pueden inferir las razones por las cuales desde el año 2010, durante el Gobierno Santos, la medición de la pobreza se hace mediante un índice compatible con la teoría de las capacidades y desarrollo humano de Sen (1981), llamada Índice de Pobreza Multidimensional, que se entiende de la siguiente manera:

\footnotetext{
La medición de la pobreza se hace tradicionalmente de forma directa e indirecta, siguiendo la clasificación de Amartya Sen (1981). El método directo evalúa los resultados de satisfacción (o no privación) que tiene un individuo respecto a ciertas características que se consideran vitales como salud, educación, empleo, entre otras. La medición indirecta evalúa la capacidad de adquisición de bienes y servicios que tienen los hogares. (DANE, 2018, p. 2)
}

Continuando con el aspecto rural, se resalta en el Acuerdo final el nivel de desigualdad económica y social de los territorios rurales con respecto a las ciudades principales. Se hace hincapié, de nuevo, en la necesidad de hacer acciones con los planes nacionales para la RRI. Una de las variables para superar la pobreza se relaciona con la producción agropecuaria. Si no hay un trabajo responsable con la implementación de los acuerdos, se podría poner en riesgo la "vida digna" de las comunidades. En este ámbito del sector rural, también se pone como foco de inseguridad y amenaza para la persona y las comunidades los cultivos ilícitos, no solo por la presencia de grupos armados ilegales dedicadas al narcotráfico a causa de la presencia débil o nula de las instituciones del Estado, sino por el impacto ambiental que el proceso de estos cultivos puede generar.

Otros puntos de seguridad humana que se encuentran en el documento son la promoción de la educación formal y la educación para el trabajo y el desarrollo humano; de vivienda; de cultura, recreación y deporte; de protección y recuperación del medio ambiente. De nuevo, uno de los puntos importantes de la seguridad humana es la protección y recuperación del medio ambiente. Esto es compatible con las indicaciones de las Naciones Unidas que desde el año 1994 consideró 
los daños ambientales como las nuevas amenazas que se deben contrarrestar mediante acciones de prevención, recuperación y preservación del medio ambiente. En otras palabras, los daños ambientales generan impactos sobre aspectos propios de la seguridad humana.

En general, como lo afirma el mismo Acuerdo final, la seguridad debe entenderse como un valor democrático y bajo la perspectiva del humanismo que deben inspirar las formas de acción por parte del Estado.

\section{Categoría de análisis: paz territorial}

El Acuerdo final tiene un enfoque territorial que consiste en "tener en cuenta las necesidades, características y particularidades económicas, culturales y sociales de los territorios y las comunidades, garantizando la sostenibilidad socio-ambiental; y procurar implementar las diferentes medidas de manera integral y coordinada, con la participación activa de la ciudadanía" (Acuerdo final, 2016, p. 6). Aquí se pueden distinguir tres elementos importantes: 1) cada territorio tiene sus particularidades y necesidades, por tanto, las exigencias e implementación de los acuerdos deben ser diferenciales; 2) se insiste en la protección y sostenibilidad socio-ambiental en que se pueda tener un equilibrio entre la vida digna de los individuos, pero sin que esto implique un daño ambiental; 3) la participación ciudadana en el momento de implementar los planes de desarrollo.

Dentro del ámbito de la seguridad, en el documento se contempla la presencia del Estado, que deberá ser amplia y eficaz, además orientada al cumplimiento de los derechos de todos en democracia. Otra forma de paz territorial del Acuerdo final es la que implica regularizar y proteger los derechos de la propiedad rural como una forma de garantizar los derechos de las personas a que sean dueñas y legítimas poseedoras de sus tierras. El cumplimiento de la seguridad territorial podría garantizar no volver a recurrir al conflicto armado para solucionar dicho problema.

Por otra parte, el documento menciona la necesidad de fortalecer la presencia del Estado en los territorios afectados por el cultivo ilícito no solamente en temas de seguridad contra las organizaciones criminales, sino también promoviendo el desarrollo integral y la satisfacción de los derechos de todas las personas:

Fortalecer la presencia institucional del Estado en los territorios afectados por los cultivos de uso ilícito, promoviendo el desarrollo integral y la satisfacción de los derechos de todos los ciudadanos y las ciudadanas; garantizando la seguridad, la convivencia y la observancia y protección de los derechos humanos; y asegurando la provisión de infraestructura, servicios públicos, educación, acceso a las comunicaciones, entre otros, de manera que se asegure el respeto y aplicación de los principios y normas del Estado Social de Derecho. (Acuerdo final, 2016, p. 105) 
La presencia que se reclama es la de sus instituciones políticas, policivas y sociales, pues su ausencia podría desencadenar otro tipo de violencia a causa del negocio del narcotráfico y la existencia de otros grupos armados ilegales.

\section{Conclusiones}

Desde la firma del Acuerdo final en noviembre de 2016 hasta el año 2019, es decir, en 3 años de implementación, se han vistos avances, no muy significativos y lentos, respecto a los puntos relacionados con la seguridad humana, el medio ambiente y la paz territorial. Tanto desde el Instituto Kroc, en su evaluación de 2 años de implementación, como de las Naciones Unidas, se pueden inferir 3 conclusiones al respecto: primero, que si bien existe voluntad política del Gobierno para implementar los compromisos, aún no es suficiente porque faltan más acciones, por ejemplo, como no recortar el presupuesto para las entidades encargadas del RRI, mantener la erradicación manual de cultivos ilícitos y avanzar en los temas legislativos; segundo, la persistencia de economías ilegales basados en la extracción de recursos naturales que afectan el medio ambiente y, en consecuencia, el buen vivir de las comunidades; tercero, la presencia aún de grupos armados ilegales en los territorios de priorización que han imposibilitado la implementación y la presencia de los instituciones políticas y sociales del Estado.

Estas dificultades, a pesar de los lentos avances en la implementación, reflejan la necesidad de pensar un tipo de seguridad centrado en la persona y las comunidades (en sentido ampliacionista). Es aquí donde está la importancia de analizar el discurso político sobre la seguridad humana en el Acuerdo final, puesto que su discurso tiene efectos pragmáticos. Cuando no existe una idea de seguridad compartida entre los actores e involucrados en la implementación, las dificultades tanto en los avances como en la promoción de la seguridad humana y la preservación y recuperación del medio ambiente podrían generar nuevas amenazas en los territorios.

La ausencia de una seguridad ampliacionista podría llevar a desencadenar otro tipo de conflictos violentos o a revivir los que han estado latentes en los territorios donde las FARC-EP estaban presentes porque los otros actores armados (Grupos Armados Organizados) desean tener el control territorial. Como se expuso en el análisis de los resultados, el Acuerdo final propone este tipo de seguridad ampliado como respuesta a una seguridad tradicional basado en los principios de la Doctrina de Seguridad Nacional (Velázquez, 2002; Sepúlveda, 2010; Ansaldi y Giordano, 2014) y la seguridad como la lucha contra el terrorismo, en el que el objeto referente ha sido el Estado. 
Por lo tanto, es evidente que el Acuerdo final haya expuesto serias preocupaciones sobre la seguridad y haya acordado poner como prioridad la securitización del medio ambiente, los territorios y los derechos económicos, culturales y políticos para garantizar el buen vivir de las personas, particularmente de aquellas que han vivido el conflicto en los territorios. Por eso, el primer punto de acuerdo es la RRI, porque desde allí se deben combatir las amenazas objetivas que han llevado a la violencia, la pobreza y la desigualdad social. Es en la RRI y, más adelante en el punto 4 sobre la erradicación y superación de los cultivos ilícitos, que se expresa mejor la idea de una seguridad humana, ambiental, y la paz territorial.

Ahora bien, es insoslayable comentar las 2 siguientes concepciones de seguridad que están explícitas en el Acuerdo final:

1) "Los protocolos de seguridad acordados se fundamentan en una concepción de la seguridad, en la cual las personas y las comunidades son su eje central". (p. 64).

2) Como parte de una concepción moderna, cualitativamente nueva, de la seguridad que, en el marco del fin del conflicto, se fundamenta en el respeto de la dignidad humana, en la promoción y respeto de los derechos humanos y en la defensa de los valores democráticos”. (p. 38)

Una seguridad centrada en la persona y las comunidades es una concepción compatible con el acuerdo de paz y se complementa con la garantía de los derechos económicos, sociales, culturales, políticos y ambientales de las comunidades. Es de esta manera como la seguridad en sentido ampliacionista se afianza a partir de 2 ideas: por un lado, centrada en las personas y comunidades; por el otro, con un enfoque en derechos. Con el fin de proteger a las comunidades y su buen vivir, el Acuerdo final considera la seguridad humana, ambiental, y la paz territorial.

Para contrarrestar las amenazas que ponen en riesgo el buen vivir de las comunidades no debe hacerse uso de la militarización (seguridad en sentido tradicional), sino a través de las instituciones políticas y sociales del Estado (sentido ampliacionista). La seguridad en sentido ampliacionista debe iniciarse en el posconflicto y perdurar en el tiempo hasta la construcción de "la paz estable y duradera", para entenderla como una política de paz. En contraste, la seguridad en sentido tradicional será necesaria pero exclusivamente durante las etapas del posconflicto para garantizar el control y presencia territorial por parte del Estado.

De este modo, la seguridad tradicional estará presente para mantener el orden público interno ante amenazas de la delincuencia común y los grupos armados ilegales vigentes, y para proteger la soberanía ante amenazas externas. En cuanto la seguridad ampliacionista, esta perdura, incluso existiendo la "paz estable y duradera". 
La siguiente tabla comprende la seguridad de las comunidades en sentido ampliacionista:

Tabla 3

Seguridad a la persona y comunidades con un enfoque ampliacionista

\begin{tabular}{llll} 
& \multicolumn{2}{c}{ Seguridad ampliacionista } \\
\hline Objeto referente & Estado de cosas a securitizar & Tipo de seguridad & Mecanismos \\
\hline \multirow{3}{*}{ Persona y comunidades } & Medio ambiente & Seguridad ambiental & $\begin{array}{l}\text { Instituciones políticas y sociales } \\
\text { durante el proceso de paz y luego } \\
\end{array}$ \\
\cline { 2 - 4 } & Derechos fundamentales y humanos & Seguridad humana & de alcanzar la "paz estable y \\
\cline { 2 - 4 } & Territorios & Paz territorial & duradera" \\
\hline
\end{tabular}

Fuente: elaboración propia.

Una seguridad en sentido ampliacionista implica que amenazas como pobreza extrema, cambio climático, minería ilegal, cultivos ilícitos, narcotráfico, desnutrición, desplazamiento forzado, que ponen en riesgo a grupos societales e individuos, deben neutralizarse con seguridad humana y ambiental, y paz territorial. Si en la implementación de los puntos estratégicos del acuerdo (1 y 4 ) el Estado pretende securitizar estas amenazas militarizándolas, podría convertirse en el generador de otro tipo de amenazas o agudizar las existentes. Esto se debe a que dichas amenazas podrían poner a las comunidades o individuos ante el par de dilemas expuestos en el marco teórico: o ante el dilema de supervivencia (desplazamiento forzado para sobrevivir) o ante el dilema de seguridad, que consiste en "matar o morir", generando, de este modo, nuevos grupos armados ilegales o el rearme de algunos desmovilizados de las FARC.

\section{Conflicto de intereses}

El autor declara la inexistencia de conflicto de interés con institución o asociación comercial de cualquier índole.

\section{Referencias}

Acuerdo final, (2017). Acuerdo final para la terminación del conflicto y la construcción de una paz estable y duradera (noviembre, 2016). https://www.cancilleria.gov.co/sites/default/files/Fotos2016/12.11_1.2016nuevoacuerdofinal.pdf 
Albrecht, U. y Brauch-Günter, H. (2009). Seguridad en la investigación para la paz y en los estudios de seguridad. En: U. Oswald Spring \& H. Günter Brauch (Comps.), Reconceptualizar la seguridad en el siglo XXI (pp. 329-373). Centro Regional de Investigaciones Multidisciplinarias. http://biblioteca.clacso.edu.ar/Mexico/crim-unam/20100329020502/ Reconceptualizarlaseguridad.pdf

Alkire, S., \& Santos, M. E. (2010). Acute Multidimensional Poverty: A New Index for Developing Countries. OPHI Working Papers, (38). University of Oxford. https://ophi.org.uk/ acute-multidimensional-poverty-a-new-index-for-developing-countries/

Ansaldi, W. y Giordano, V. (2014). América Latina. Tiempos de violencia. Editorial Ariel.

Bartolomé, M. (2006). La seguridad internacional post 11-S: Contenidos, debates y tendencias. Instituto de Publicaciones Navales del Centro Naval.

Botche, M., Bruch, C., Diamond, J. y Jensen, D. (2010). El derecho internacional y la protección del medio ambiente durante los conflictos armados: lagunas y oportunidades. International Review of Red Cross, (879), 321-346. https://international-review.icrc.org/sites/ default/files/icrc-003-0879-bothe.pdf

Brauch-Günter, H. (2009). Cuarteto conceptual: la seguridad y sus vínculos con la paz, el desarrollo y el ambiente. En: U. Oswald-Spring \& H. Günter-Brauch (Comps.), Reconceptualizar la seguridad en el siglo XXI (pp. 151-228). Centro Regional de Investigaciones Multidisciplinarias. http://biblioteca.clacso.edu.ar/Mexico/crim-unam/20100329020502/ Reconceptualizarlaseguridad.pdf

Buzan, B., \& Waever, O. (2003). Regions and Powers the Structure of International Security. Cambridge University Press.

Buzan, Wæver y Wilde (1998). Security: A New Framework for Analysis. United State of America: Lynne Rienner Publishers.

Centro Nacional de Memoria Histórica. (2016). Tierras y conflictos rurales. Historia, políticas agrarias y protagonistas. $\mathrm{CNMH}$.

Departamento Administrativo Nacional de Estadística (DANE). (2018). Índice de pobreza multidimensional en Colombia, año 2018. https:/www.dane.gov.co/files/investigaciones/condiciones_vida/pobreza/2018/bt_pobreza_multidimensional_18.pdf 
Departamento Nacional de Planeación (DNP). (2011). Plan Nacional de Desarrollo: "Prosperidad para todos", 2010-2014. DNP. https://www.dnp.gov.co/Plan-Nacional-de-Desarrollo/ PND-2010-2014

Estrada, J. (2016). Elementos para una problematización básica del Punto 3 "Fin del conflicto" y del Punto 6 "Implementación, Verificación y Refrendación" de la Agenda de diálogos de La Habana. En: S. Alvarado, E. Rueda y P. Gentili (Eds.), Paz en Colombia: perspectivas, desafíos, opciones (págs. 91-108). CLACSO.

Fairclough, N. (1995). Introducción General Introduction. En Federico Navarro (Trad.), Análisis crítico del discurso. El studio crítico del discurso. (pp. 1-20). UBA.

Fundación Paz y Reconciliación. (2015). Lo que hemos ganado. Han disminuido los combates, las muestres, los heridos, los secuestros y los desplazamientos. Fundación Paz y Reconciliación. https://e7c20b27-21c2-4f2b-9c38-a1a16422794e.usrfiles.com/ugd/e7c20b_4af15 55b9dbd4922adf0cce8bd622f36.pdf

González, C. (2015). El territorio de la paz es Colombia y su gente. https://www.semillas.org.co/ es/el-territorio-de-la-paz-es-colombia-y-su-gente

Grocio, H. (1925). Del derecho de la guerra y de la paz (J. Torrubiano Ripoll, Trad.). Ed. Reus.

Grotius, H. (1975). Prolegomena to the Law of War and Peace. The Bobbs-Merill Company Inc.

Instituto Kroc de Estudios Internacionales de Paz. (2019a). Estado efectivo de implementación del Acuerdo de Paz de Colombia 2 años de implementación (Informe 3). Kroc Institute, University of Notre Dame. https://kroc.nd.edu/assets/321729/190523_informe_3_final_ final.pdf

Instituto Kroc de Estudios Internacionales de Paz. (2019b). Actualización. Informe 3 del Instituto Kroc. Hacia una paz de calidad en Colombia. https://kroc.nd.edu/assets/315919/190408_ actualizacio_n_informe_3_instituto_kroc_feb19.pdf

Jaramillo, S. (2013). Paz territorial. Oficina del Alto Comisionado para la Paz. Presidencia de la República. https://interaktive-demokratie.org/files/downloads/La-Paz-Territorial.pdf

Londoño, M. y Martínez, L. (2015). El medio ambiente, otra víctima del conflicto armado colombiano actual. Tesis de grado Universidad de Manizales. https://studylib.es/doc/7592015/ el-medio-ambiente-otra 
Naciones Unidas. (2020). Informe anual del Alto Comisionado de las Naciones Unidas para los Derechos Humanos e informes de la Oficina del Alto Comisionado y del Secretario General. Situación de los derechos humanos en Colombia. https://www.hchr.org.co/index. php/informes-y-documentos/informes-anuales/9562-informe-de-la-alta-comisionada-delas-naciones-unidas-para-los-derechos-humanos-sobre-la-situacion-de-derechos-humanos-en-colombia-durante-el-ano-2020

Naciones Unidas y Cooperación Alemana. (2014). Construcción de una paz territorial estable, duradera y sostenible en Colombia. file://C:/Users/wilfa/Downloads/undp-co-pazyambiente-2015.pdf

Oswald-Spring, Ú. y Günter-Brauch, H. (2009). Globalización y desafíos ambientales cambian la conceptualización de seguridad en América Latina. En Ú. Oswald-Spring \& H. GünterBrauch (Comps.), Reconceptualizar la seguridad en el siglo XXI (págs.. 37-70). Centro Regional de Investigaciones Multidisciplinarias. http://biblioteca.clacso.edu.ar/Mexico/ crim-unam/20100329020502/Reconceptualizarlaseguridad.pdf

Programa de las Naciones Unidas para el Desarrollo (PNUD). (2010). Informe sobre desarrollo humano. La verdadera riqueza de las naciones: Caminos del desarrollo humano. http://hdr. undp.org/sites/default/files/hdr_2010_es_complete_reprint.pdf

Rodríguez-Rodríguez, D. (2017). La paz ambiental: retos y propuestas para el posacuerdo. Centro de Estudios de Derecho, Justicia y Sociedad (Dejusticia). https://www.dejusticia.org/ wp-content/uploads/2017/04/fi_name_recurso_924.pdf

Sepúlveda, A. (2010). América Latina. Militares y política después del fin de la Guerra Fría. En M. Bartolomé (Comp.), Seguridad y defensa en tiempos del Bicentenario. Visiones desde Argentina y Chile (págs. 305-320. Instituto de Publicaciones Navales del Centro Naval.

United Nations Environment Programme (UNEP). (2009). From Conflict to Peace Building: The Role of Natural Resources and the Environment. UNEP. https://postconflict.unep.ch/publications/pcdmb_policy_01.pdf

United Nations (UN). (1986). Concepts of Security. UN (Disarmament Study Series 14). https:// digitallibrary.un.org/record/99705

Valencia, L. y Obando, C. (2015). Retos nacionales y territoriales del posconflicto. En lo que hemos ganado Bogotá: Fundación Paz y Reconciliación. 
Van Dijk, T. (1999a). ¿Qué es análisis del discurso político? En T. Dijk \& I. Mendizábal, Análisis del discurso social y político (págs. 9-102). ABYA-YALA. https://digitalrepository.unm. edu/cgi/viewcontent.cgi?referer $=\&$ httpsredir $=1 \&$ article $=1414 \&$ context=abya_yala

Van Dijk, T. (1999b). El análisis crítico del discurso. Anthropos, (186), 23-36. http://www.discursos.org/oldarticles/El\%20an\%E1lisis\%20cr\%EDtico\%20del\%20discurso.pdf

Van Dijk, T. (2003). La multidisciplinaridad del análisis crítico del discurso: un alegato en favor de la diversidad. En R. Wodak y M. Meyer (compiladores), Métodos de análisis crítico del discurso (págs. 143-177), Barcelona: Gedisa.

Velásquez Rivera, Édgar. (2017). Historia de la Doctrina de la Seguridad Nacional. Estudios Latinoamericanos, (14-15), 74-82. Recuperado a partir de https://revistas.udenar.edu.co/index.php/rceilat/article/view/3279

Wæver, O. (2009). Paz y seguridad: dos conceptos en evolución y su relación cambiante. En U. Oswald-Spring \& H. Günter-Brauch (Comps.), Reconceptualizar la seguridad en el siglo XXI (pp. 329-373). Centro Regional de Investigaciones Multidisciplinarias. http://biblioteca.clacso.edu.ar/Mexico/crim-unam/20100329020502/Reconceptualizarlaseguridad.pdf

Wolfers, A. (1962). National Security as an Ambiguous Symbol. En Wolfers, A., Discord and Collaboration. Essays on International Politics (pp. 147-165). John Hopkins University Press. 